\title{
Hadronic beam models for quasars and microquasars
}

\author{
D. F. Torres ${ }^{1}$ and A. Reimer ${ }^{2}$ \\ ${ }^{1}$ ICREA \& Institut de Ciències de l'Espai (IEEC-CSIC), Campus UAB, Facultat de Ciències, Torre C5-parell, 2a planta, \\ 08193 Barcelona, Spain \\ e-mail: dtorres@ieec.uab.es \\ ${ }^{2}$ Institut für Theoretische Physik, and Institut für Astro- und Teilchenphysik, Leopold-Franzens-Universität Innsbruck, \\ Technikerstr. 25, 6020 Innsbruck, Austria
}

Received 10 January 2011 / Accepted 2 February 2011

\begin{abstract}
Context. Most of the hadronic jet models for quasars (QSOs) and microquasars (MQs) found in the literature represent beams of particles (e.g. protons). These particles interact with the matter in the stellar wind of the companion star in the system or with crossing clouds, generating $\gamma$-rays via proton-proton processes.

Aims. Our aim is to derive the particle distribution in the jet as seen by the observer, so that the $\gamma$-ray and neutrino yields can be properly computed.

Methods. We use relativistic invariants to obtain the transformed expressions in the cases of both a power law and power law with a cutoff particle distribution in the beam. We compare them with previous expressions used earlier in the literature.

Results. We show that formerly used expressions for the particle distributions in the beam as seen by the observer are in error, with the differences being strongly dependent on the viewing angle. For example, for $\Gamma=10$ ( $\Gamma$ is the Lorentz factor of the blob) and angles larger than $\sim 20^{\circ}$, the calculation used earlier entails an overprediction (order of magnitude or more) of the proton spectra for $E>\Gamma m c^{2}$, whereas it always overpredicts (two orders of magnitude) the proton spectrum at lower energies, for all the viewing angles. Conclusions. All the results for photon and neutrino fluxes in hadronic models are affected in beams that have made use of the earlier calculation. Given that correct $\gamma$-ray fluxes will in almost any case be significantly diminished in comparison with published results and that the time of observations in Cherenkov facilities grows with the square of the flux-reduction factor in a statistically limited result, the possibility of observing hadronic beams is undermined.
\end{abstract}

Key words. ISM: jets and outflows - astroparticle physics - Gamma rays: general

\section{Introduction}

The study of the possible high-energy radiation from QSOs and MQs is uncertain in one central aspect, the particle composition of the jets. Is the radiation emitted produced mainly via inverse Compton (self-synchrotron and/or with external fields) or via proton-proton/proton-photon interactions leading to subsequent meson decay? In the latter case, hadronic jet models have been used to assess this possibility. Most of these models represent beams of particles (e.g. protons) linearly propagating in a direction normal to an accretion disk, interacting with the matter in the stellar wind of the companion star in the case of MQs or with matter in clouds in the case of quasars. The protons in the beams are usually assumed to be distributed with a power law that may be cut at high energies, as is the case when they were accelerated in Fermi processes within the jet and subject to losses. It is then crucial to have a correct description of the proton distribution as seen by the observer, since the emissivity of $\gamma$-rays and neutrinos produced by charged and neutral pions make use of the cross sections known in that frame. In this paper we present the derivation of a beam particle distribution as seen by the observer and compare it with the expression used earlier, finding significant differences. Consequences are discussed.

\section{Derivation of the particle distribution}

In what follows, primed quantities refer to quantities that reside in the jet frame, whereas unprimed quantities are used for those in the observer frame. We consider the Lorentz transformations for energies and momenta

$$
\begin{aligned}
E & =\Gamma\left(E^{\prime}+\beta c p_{\|}^{\prime}\right), \quad E^{\prime}=\Gamma\left(E-\beta c p_{\|}\right), \\
p_{\|} & =\Gamma\left(p_{\|}^{\prime}+\beta E^{\prime} / c\right), \quad p_{\|}^{\prime}=\Gamma\left(p_{\|}-\beta E / c\right),
\end{aligned}
$$

where $p_{\|}=p \cos (\theta)$ and $p_{\perp}=p \sin (\theta)$ are the parallel and perpendicular momentum, respectively. For the relativistic case, $m c \ll p$ and $m c^{2} \ll E$, the transformation of the energy simplifies to $E=D E^{\prime}$, with $D=1 /[\Gamma(1-\beta \cos (\theta))]$.

The elementary invariants under Lorentz transformations are (see, e.g., Dermer \& Menon 2010) the invariant four-volume, $\mathrm{d} \boldsymbol{x} \mathrm{d} t=\mathrm{d} V \mathrm{~d} t$; the invariant phase-space element, $\mathrm{d} \boldsymbol{p} / E=$ $p^{2} \mathrm{~d} p \mathrm{~d} \Omega / E \rightarrow \epsilon \mathrm{d} \epsilon \mathrm{d} \Omega$, with the final expression applying to photons and extremely relativistic particles; and the invariant phase volume, $\mathrm{d} v=\mathrm{d} \boldsymbol{p} \mathrm{d} \boldsymbol{x}$, where the boldface stands for threedimensional space magnitudes. Because the number $N$ of particles or photons is invariant, one finds that

$$
\frac{\mathrm{d} N}{\mathrm{~d} v}=\frac{1}{p^{2}} \frac{\mathrm{d} N}{\mathrm{~d} V \mathrm{~d} p \mathrm{~d} \Omega}
$$


is also invariant. For photons and extremely relativistic particles, the last invariant becomes

$\frac{1}{\epsilon^{2}} \frac{\mathrm{d} N}{\mathrm{~d} V \mathrm{~d} \epsilon \mathrm{d} \Omega}$

Using the equality $E^{2}=c^{2} p^{2}+m^{2} c^{4}$ allows writing $p^{2} \mathrm{~d} p=$ $\left(p E / c^{2}\right) \mathrm{d} E$. Using the latter in Eq. (2) implies that the expression

\section{$\frac{1}{p E} \frac{\mathrm{d} N}{\mathrm{~d} V \mathrm{~d} E \mathrm{~d} \Omega}$}

is invariant under Lorentz transformations. Again, for photons and extremely relativistic particles, $p c=E$, $(=\epsilon$, in the notation above for this case), and the last invariant becomes expression (3). We define the differential number density of particles as $n(E, \Omega) \equiv \mathrm{d} N /[\mathrm{d} V \mathrm{~d} E \mathrm{~d} \Omega]$, i.e., the differential number of photons or relativistic particles with dimensionless energy between $E$ and $E+\mathrm{d} E$ that are directed into differential solid angle interval $\mathrm{d} \Omega$ in the direction $\Omega$ of some physical volume $\mathrm{d} V$. Because of invariant (4) and the definition of $n(E, \Omega)$, we can write

$n(E, \Omega)=n^{\prime}\left(E^{\prime}, \Omega^{\prime}\right)\left(\frac{p}{p^{\prime}}\right)\left(\frac{E}{E^{\prime}}\right)$

which is valid in general, and

$n(E, \Omega)=n^{\prime}\left(E^{\prime}, \Omega^{\prime}\right)\left(\frac{E^{2}}{E^{\prime 2}}\right)$

which is valid for photons and extremely relativistic particles. Equation (5) just says that the expression for the distribution function $f(\boldsymbol{r}, \boldsymbol{p})$ appearing in kinetic theory of gases, such that the product $f(\boldsymbol{r}, \boldsymbol{p}) \mathrm{d} \boldsymbol{p} \mathrm{d} V$ is the number of particles lying in a given volume element $\mathrm{d} V$ and having momenta in definite intervals d $\boldsymbol{p}$, is Lorentz-invariant (see e.g., Eq. (10.5) of Landau \& Lifshitz 1987). We note that

$f(\boldsymbol{r}, \boldsymbol{p})=\frac{\mathrm{d} N}{\mathrm{~d} V \mathrm{~d} \boldsymbol{p}}=\frac{\mathrm{d} N}{\mathrm{~d} V p^{2} \mathrm{~d} p \mathrm{~d} \Omega}=\frac{\mathrm{d} N}{\left(p E / c^{2}\right) \mathrm{d} V \mathrm{~d} E \mathrm{~d} \Omega}$,

and the invariance of $f(\boldsymbol{r}, \boldsymbol{p})$ is the one expressed by Eq. (4).

\subsection{The extremely relativistic case}

In this case, the particle density in the jet frame transforms into the observer frame, if it is a power law defined as

$n^{\prime}\left(E^{\prime}, \Omega^{\prime}\right)=(A / 4 \pi) E^{\prime-\alpha}$,

by virtue of the previous formulae ((6) and (8)), as

$$
\begin{aligned}
n(E, \Omega) & =\left(E / E^{\prime}\right)^{2} n^{\prime}\left(E^{\prime}, \Omega^{\prime}\right)=(A / 4 \pi) D^{2}(E / D)^{-\alpha} \\
& =(A / 4 \pi) E^{-\alpha} D^{2+\alpha} .
\end{aligned}
$$

\subsection{The general case}

Using Eqs. (1) and the one for $E^{2}$, it is possible to find that

$$
\begin{aligned}
E / E^{\prime} & =E /[\Gamma(E-\beta c p \cos (\theta))] \\
& =\frac{1}{\Gamma\left(1-\beta \cos (\theta) \sqrt{1-m^{2} c^{4} / E^{2}}\right)} .
\end{aligned}
$$

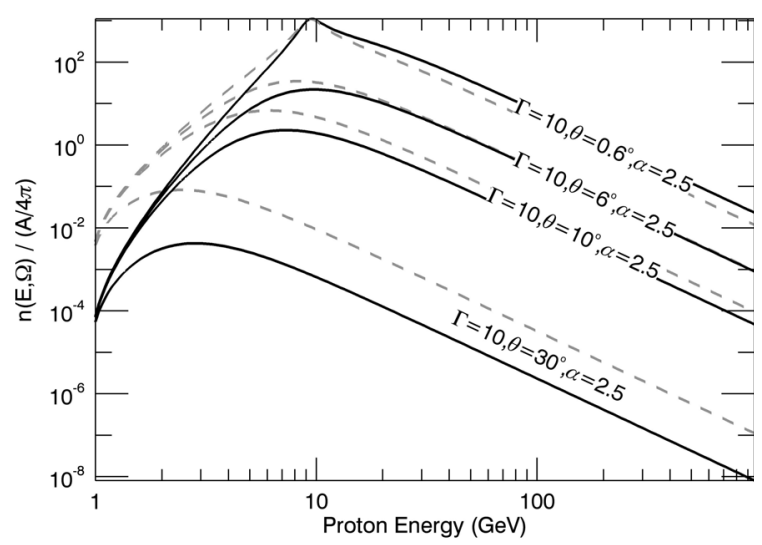

Fig. 1. A power-law spectrum of protons in a beam as seen by the observer for different values of viewing angles, and an example of Lorentz factor and slope (solid lines). The gray dashed lines stand for the earlier derivation results for the same parameters (see Sect. 2.4).

In addition, using the Lorentz transformation for momenta (Eq. (1) and that $p_{\perp}=p \sin (\theta)=p_{\perp}^{\prime}$ ), one has

$$
\begin{aligned}
p^{\prime 2} & \left.=p^{2} \sin ^{2}(\theta)+\Gamma^{2}(p \cos (\theta)-\beta E / c)^{2}\right) \\
& =p^{2}\left[\sin ^{2}(\theta)+\Gamma^{2}\left(\cos (\theta)-\beta E / \sqrt{E^{2}-m^{2} c^{4}}\right)^{2}\right] .
\end{aligned}
$$

Using the latter expression to get $p / p^{\prime}$ and inserting it into Eq. (5), together with Eqs. (8) and (10) we get,

$$
\begin{aligned}
n(E, \Omega)= & \frac{A}{4 \pi} \\
& \times \frac{\Gamma^{-\alpha-1} E^{-\alpha}\left(1-\beta \cos (\theta) \sqrt{1-m^{2} c^{4} / E^{2}}\right)^{-\alpha-1}}{\left[\sin ^{2}(\theta)+\Gamma^{2}\left(\cos (\theta)-\frac{\beta}{\sqrt{1-m^{2} c^{4} / E^{2}}}\right)^{2}\right]^{1 / 2}} .
\end{aligned}
$$

In the limit when $m c^{2} \ll E$, the last expression reduces to $(A / 4 \pi) E^{-\alpha} D^{2+\alpha}$. To prove this we use $\left(\sin ^{2}(\theta)+\Gamma^{2}(\cos (\theta)-\beta)^{2}\right)^{1 / 2}=1 / D$, which can be established by squaring both sides, and then by summing and subtracting $\Gamma^{2} \sin ^{2}(\theta)+\Gamma^{2} \beta^{2} \cos ^{2}(\theta)$ on the lefthand side, with additional use of the definition of $\Gamma^{2}=1 /\left[1-\beta^{2}\right]$. Also we do not assume a specific jet composition, so it is a general result for any kind of particle distributions. Figure 1 shows the resulting expression (12) for a power-law spectrum of protons in a beam as seen by the observer, for different values of viewing angles, and an example of Lorentz factor and slope (solid lines). For high energies, when $E>\Gamma m c^{2}, n(E, \Omega)$ is a power-law distribution, as the original primed one, albeit it presents a different behavior for lower values of $E$.

\subsection{A power law with an exponential cutoff}

We also briefly consider the case in which the intrinsic particle distribution in the beam is a power law with an exponential cutoff. This would be a natural consequence of particles being subject to losses on the same site as where they are accelerated. As such, the cutoff appears in the primed referenced frame, where

$n^{\prime}\left(E^{\prime}, \Omega^{\prime}\right)=(A / 4 \pi) E^{\prime-\alpha} \exp \left(-E^{\prime} / E_{\text {cut }}\right)$.

Here, $E_{\text {cut }}$ should be fixed in the beam frame, where acceleration and losses are supposed to occur, but once fixed it is not subject 
to Lorentz transformations. However, the energy variable in the exponential function is. The result is

$$
\begin{aligned}
n(E, \Omega)= & \frac{A}{4 \pi} \\
& \times \frac{\Gamma^{-\alpha-1} E^{-\alpha}\left(1-\beta \cos (\theta) \sqrt{1-m^{2} c^{4} / E^{2}}\right)^{-\alpha-1}}{\left[\sin ^{2}(\theta)+\Gamma^{2}\left(\cos (\theta)-\frac{\beta}{\sqrt{1-m^{2} c^{4} / E^{2}}}\right)^{2}\right]^{1 / 2}} \\
& \times \exp \left(-\frac{\Gamma E}{E_{\mathrm{cut}}}\left(1-\beta \cos (\theta) \sqrt{1-m^{2} c^{4} / E^{2}}\right) .\right.
\end{aligned}
$$

When $m c^{2} \ll E$, the exponential cutoff $\exp \left(-E^{\prime} / E_{\text {cut }}\right)$ is transformed into $\exp \left(-E / D E_{\text {cut }}\right)$, with $D$ the bulk Doppler factor.

\subsection{Comparison with Purmohammad \& Samimi (2001)}

Purmohammad \& Samimi (2001, hereafter PS01) state that the isotropic particle distribution transformed from the jet into the observer frame results in (in this section and to avoid confusion we label their expressions with $\star)$ :

$$
\begin{aligned}
n_{\star}(E, \Omega)= & \frac{A}{4 \pi} \Gamma^{-\alpha+1} E^{-\alpha} \\
& \times \frac{\left(1-\beta \cos (\theta) \sqrt{1-m^{2} c^{4} / E^{2}}\right)^{-\alpha}}{\left[\sin ^{2} \theta+\Gamma^{2}\left(\cos (\theta)-\frac{\beta}{\sqrt{1-m^{2} c^{4} / E^{2}}}\right)^{2}\right]^{1 / 2}} .
\end{aligned}
$$

Purmohammad \&Samimi's (2001) paper does not provide a derivation of their Eq. (3), quoted above as (15), but earlier private communication with one of us was sufficient to reconstruct it, as follows. They start by assuming conservation of particles in the form

$$
n_{\star}(E, \Omega) \mathrm{d} V \mathrm{~d} \Omega \mathrm{d} E=n_{\star}^{\prime}\left(E^{\prime}, \Omega^{\prime}\right) \mathrm{d} V^{\prime} \mathrm{d} \Omega^{\prime} \mathrm{d} E^{\prime},
$$

thus one has

$n_{\star}(E, \Omega)=n_{\star}^{\prime}\left(E^{\prime}(E, \theta), \Omega^{\prime}(E, \theta)\right) \frac{\mathrm{d} V^{\prime}}{\mathrm{d} V} \frac{\mathrm{d} \Omega^{\prime}}{\mathrm{d} \Omega} \frac{\mathrm{d} E^{\prime}}{\mathrm{d} E}$.

From the Lorentz-invariance of the phase-space element $\mathrm{d} \boldsymbol{p} / E$, the invariance of $p \mathrm{~d} \Omega \mathrm{d} E$ can also be proved. Then, one can write

$$
\frac{p}{p^{\prime}}=\frac{\mathrm{d} E^{\prime}}{\mathrm{d} E} \frac{\mathrm{d} \Omega^{\prime}}{\mathrm{d} \Omega}
$$

Using the expressions derived above for $p / p^{\prime}$ and the equality $\mathrm{d} V^{\prime}=\Gamma \mathrm{d} V$ into (17), one gets

$$
n_{\star}(E, \Omega)=\frac{\Gamma(A / 4 \pi) E^{\prime-\alpha}}{\sqrt{\sin ^{2}(\theta)+\Gamma^{2}\left(\cos (\theta)-\beta E / \sqrt{E^{2}-m^{2} c^{4}}\right)^{2}}} .
$$

From here, after replacing in the numerator $E^{\prime}=\Gamma(E-$ $\beta c p \cos (\theta))=\Gamma\left(E-\beta \cos (\theta) \sqrt{E^{2}-m^{2} c^{4}}\right)$, expression (15) follows immediately.

Formally, one can say that there is a problem hidden in Eq. (16), from which the rest of the derivation follows. The invariant phase volume is not $\mathrm{d} V \mathrm{~d} \Omega \mathrm{d} E$ but rather $p^{2} \mathrm{~d} V \mathrm{~d} \Omega \mathrm{d} p$. To avoid inconsistencies, invariants (from which to derive transformation laws) should be written as operations over individually invariant factors, e.g., $\mathrm{d} N$ over $\mathrm{d} v$, rather than from identities (which is what one obtains by replacing the definition of $n$ and $n^{\prime}$ in Eq. (16)). However, the latter can also yield the correct result, too, if care is exercised. In practice, then, the problem with the prior derivation is in the use of the equality $\mathrm{d} V^{\prime}=\Gamma \mathrm{d} V$. This equation implies that $\mathrm{d} V^{\prime}$ is the proper volume $\mathrm{d} V_{0}$, which is clearly incorrect, given that the momentum, $\boldsymbol{p}^{\prime}$, of the particles in that volume is not zero. Only for the proper volume is the referred equality true (see, e.g., Eq. (4.6) of Landau \& Lifshitz 1987). To clarify this issue, let us introduce, in addition of the two reference systems (observer and beam), another frame $K_{0}$ in which the particles with the given momentum are at rest. The proper volume $\mathrm{d} V_{0}$ of the element occupied by the particles is defined relative to this system. The velocities of the primed and unprimed systems relative to the system $K_{0}$ coincide by definition with the velocities $v$ and $v^{\prime}$, which these particles have in the systems of the observer and the beam, respectively. Thus, we can write

$$
\mathrm{d} V=\mathrm{d} V_{0} \sqrt{1-\mathrm{v}^{2} / c^{2}}, \quad \mathrm{~d} V^{\prime}=\mathrm{d} V_{0} \sqrt{1-\mathrm{v}^{\prime 2} / c^{2}}
$$

from which one can write

$\frac{\mathrm{d} V}{\mathrm{~d} V^{\prime}}=\frac{\sqrt{1-\mathrm{v}^{2} / c^{2}}}{\sqrt{1-\mathrm{v}^{\prime 2} / c^{2}}}=\frac{E^{\prime}}{E}$

If we use Eqs. (18) and (21) in Eq. (16), the last transforms back into the expression of the invariant quantity $1 /(p E) \times$ $\mathrm{d} N /(\mathrm{d} V \mathrm{~d} E \mathrm{~d} \Omega)$ and Eq. (5) follows.

The difference between expression (15) and our Eq. (12) is important. Dividing one by another, we obtain

$\frac{n(E, \Omega)}{n_{\star}(E, \Omega)}=\Gamma^{-2}\left(1-\beta \cos (\theta) \sqrt{1-m^{2} c^{4} / E^{2}}\right)^{-1}$,

which is a non-trivially dependent function of $\Gamma, E$, and $\theta$. However, it is not a function of $\alpha$. In the extremely relativistic case $m c^{2} \ll E$, one notices a factor $D / \Gamma$ of difference. Put otherwise, in the stated asymptotic regime, the ratio expressed by Eq. (22) reduces to $\left(n(E, \Omega) / n_{\star}(E, \Omega)\right)=\Gamma^{-2}(1-$ $\left.\cos (\theta) \sqrt{1-1 / \Gamma^{2}}\right)^{-1}$, which is only a function of $\Gamma$ and $\theta$. One can see that, in the case of $\theta=0^{\circ}, n(E, \Omega) / n_{\star}(E, \Omega) \rightarrow 2$ for growing $\Gamma$. For other values of $\theta$, this $2 \mathrm{D}$ function quickly falls off. We plot the comparison between our derivation and the one by PS01 in Fig. 2, both as a function of viewing angle $\theta$ (for fixed $\Gamma=10$ ) and bulk Lorentz factor $\Gamma$ (for $\theta=20^{\circ}$ ). We see that the differences range from a factor of $\sim 2$ in the case of directly-pointing beams of blazars and microblazars (of very low $\theta$, in our example, $0.6^{\circ}$ ), to one order of magnitude at least for the more common cases of moderate inclinations (e.g., for $20^{\circ}$; with the difference being significantly larger for larger inclinations). As soon as $\theta$ is larger than $\sim 5^{\circ}$, the proton spectrum in the beam increasingly undershoots PS01's calculation. This leads to an analogous correction of previously calculated $\gamma$-ray and neutrino emission (typically by more than one order of magnitude) in all cases in which expression (15) was used. In the low energy decline, PS01's calculation always overestimates (by about two orders of magnitude) the correct yield of the proton spectrum (see Fig. 1 at proton energies between 1 and $10 \mathrm{GeV}$ ).

\section{Discussion}

The proton-beam model presented by PS01 gives an expression for a power-law proton spectrum in a beam, as seen by the observer. We have demonstrated that this expression is incorrect, since it does not comply with relativistic invariants. We derived 

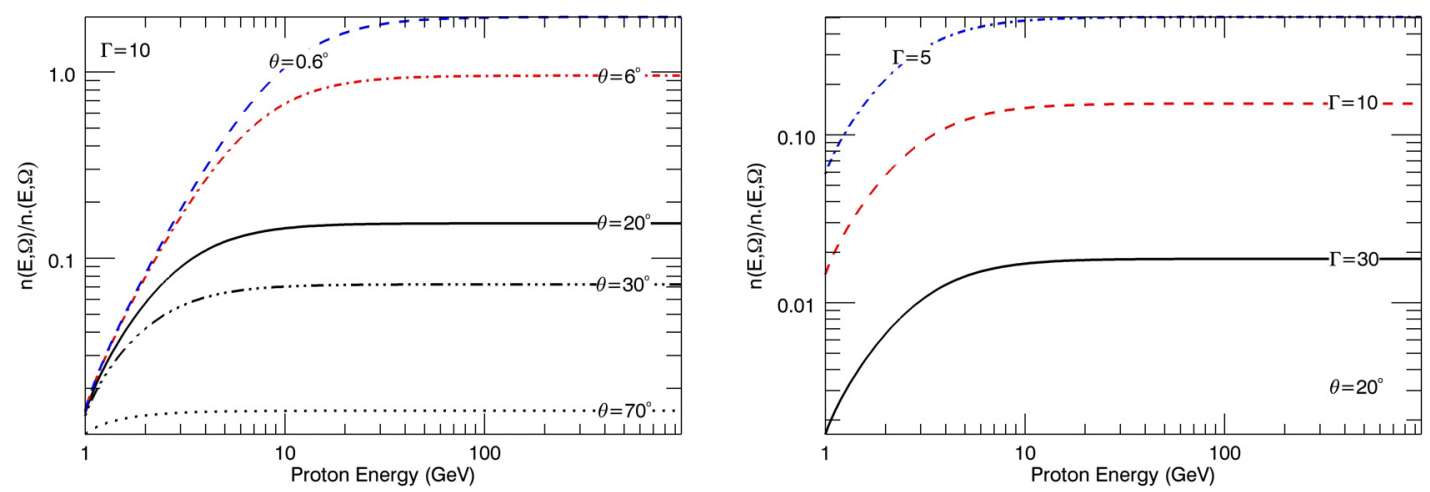

Fig. 2. Comparison of the derived expression for the spectrum of protons in beams, following Eq. (22). Left: a function of the viewing angle $(\theta)$ for $\Gamma=10$. Right: a function of the bulk Lorentz factor $(\Gamma)$, for $\theta=20^{\circ}$.

the correct transformation from the jet frame particle density to the observer's, which strictly obeys these Lorentz invariance constraints. By comparing our result with PS01, we noticed that differences depend strongly on the viewing angle. For many cases, PS01 calculation entails a significant overprediction (order of magnitude) of the proton spectra for $E>\Gamma m c^{2}$, whereas it always significantly overpredicts (two orders of magnitude) the proton spectrum at lower energies, for all viewing angles. The $\gamma$-ray luminosity is related to the particle density through the corresponding emissivity of the processes by which the particles interact. For instance, the charged and neutral pion decay channels are the most effective yield of photons and neutrinos in many situations, and its emissivity is directly proportional to the particle density $n(E, \Omega)$. Many publications that rely on the calculation by PS01 for estimating $\gamma$-ray and neutrino emission yields in hadronic beam scenarios are thus affected in this way.

One particular example can be seen in the hadronic MQ case (Romero et al. 2003) ${ }^{1}$ and in their applications (e.g., Romero et al. 2005; Orellana et al. 2007; Reynoso et al. 2008). These are all affected by our results. MQs can have $\Gamma \sim 2$, but $\theta$-values above $10^{\circ}$ are typical. MQs can precess, as for SS433, where $\theta$ can even change up to $20^{\circ}$ around an already large mean viewing angle.

Only a small part of the $\gamma$-ray AGNs are not blazars. The majority among those associated with radio sources possess relatively large core dominance (Abdo et al. 2010), indicating that their viewing angle is probably not large. Assuming $\theta<20^{\circ}$, if $\Gamma<10$, PS01 deviation is no more than a factor of $\sim 6$ (at $E>m c^{2}$ ). For the average blazar with $\Gamma=10$ and $\theta<10^{\circ}$ the difference is a factor of $\sim 2$. However, it has been argued that during the extreme TeV flaring events of some blazars (e.g. Mkn 501, PKS 2155-304), Lorentz factors greater than $\Gamma \sim 50$ are required (e.g. Begelman et al. 2008). If the viewing angle remains at $\theta \sim 1 / \Gamma_{\text {quiet }} \sim 1 / 10 \sim 5.7^{\circ}$ during such events, PS01 formulation error can grow to more than an order of magnitude. Furthermore, a few radio galaxies with large viewing angles are also detected at $\gamma$-ray energies: M87 with viewing angle $\theta<\sim 40^{\circ}$, and Cen A where estimates for the viewing angle range from $15^{\circ}$ to $80^{\circ}$. These radio galaxies have been already modeled with the PS01 derivation (e.g., Reynoso et al. 2010). Here, the difference between the PS01 formula and ours can reach a factor 20 for $\Gamma$ not larger than 5 .

Finally, we note that models designed to predict future detections of large-viewing angle sources with higher sensitivity instruments than currently available will strongly overpredict the number of sources if the PS01 formula is used. Similarly, a model for an AGN that overestimates the $\gamma$-ray flux could make wrong associations of unidentified $\gamma$-ray sources. Given that $\gamma$ ray fluxes will be diminished in many cases, and that the time of observations in Cherenkov facilities grows with the square of the flux-reduction factor, the likelihood of observing hadronic beams is undermined.

Acknowledgements. D.F.T. acknowledges support from grants AYA200907391, SGR2009-811, and TW2010005. A.R. acknowledges support by the Marie Curie IRG grant 248037 within the FP7 Program. We acknowledge O. Reimer for discussions.

\section{References}

Abdo, A., Ackermann, M., Ajello, M., et al. 2010, ApJ, 720, 912

Begelman, M., Fabian, A. C., \& Rees, M. J. 2008, MNRAS, 384, L19

Dermer, C., \& Menon, G. 2010, High-Energy Radiation from Black Holes (Oxford University Press)

Landau, L., \& Lifshitz, E. M. 1987, Theory of Fields, fourth revised English edn. (Buttenworth-Heinemann)

Purmohammad, D., \& Samimi, J. 2001, A\&A, 371, 61 (PS01)

Orellana, M., Bordas, P., Bosch-Ramon, V., Romero, G. E., \& Paredes, J. M. 2007, A\&A, 476, 9

Romero, G. E, Torres, D. F., Kaufman-Bernadó, M. M., \& Mirabel, I. F. 2003, A\&A, 410, L1

Romero, G. E, Christiansen, H. R., \& Orellana, M. 2005, APJ, 632, 1093

Reynoso, M. M., Romero, G. E., \& Christiansen, H. R. 2008, MNRAS, 387, 1745

Reynoso, M. M., Medina, M. C., \& Romero, G. E. 2010, A\&A, submitted [arXiv: $1005.3025 \mathrm{v} 1]$

\footnotetext{
1 Note that the Eq. (2) in the latter paper has a missing square in the denominator factor $\left.\left(\cos (\theta)-\beta E / \sqrt{(} E^{2}-m^{2} c^{4}\right)\right)$. This error has been adopted in several of the papers quoting Romero et al. (2003).
} 